\title{
Functional Cellulose by Microwave Initiated Grafting Polymerization in Homogeneous Media
}

\author{
Gang Chang ${ }^{1, a}$, Tiaokun Fu ${ }^{1, b}$, Xiaoyi Wei ${ }^{1, c^{*}}$, Jihua Li ${ }^{1, d}, F e i$ Wang ${ }^{1, e}$, Haiyan \\ Feng ${ }^{2, f}$
}

${ }^{1}$ Key laboratory of Tropic Crop Products Processing of Ministry of Agriculture, Agriculture Products Processing Research Institute, Chinese Academy of Tropical Agricultural Sciences, Zhanjiang 524001, China

\section{${ }^{2}$ South Subtropical Crops Research Institute, Chinese Academy of Tropical Agricultural Sciences, Zhanjiang 524091,China}

Keywords: bagasse cellulose;homogeneous grafting; microwave;characterization

Abstract. The cellulose grafted copolymer was synthesized by homogeneous media grafting 2-(Dimethylamino) ethylmethacrylate (DMAEMA) onto the sugarcane bagasse under microwave irradiation. A homogeneous solution formed with cellulose dissolved in 1-butyl-3-methylinidazolium chloride ionic liquid which the grafting polymerization was initiated by potassium persulfate in the presence of $\mathrm{N}, \mathrm{N}^{\prime}$-methylenebisacrylamide as a crosslinker. Then the crosslinking agent concentration, concentration of initiator, monomer content, temperature and time factors were investigated during the synthesization, which ultimately determined the optimal reaction conditions. It would provide the basic theory for bagasse cellulose grafting

\section{Introduction}

Cellulose itself has a good processability, thermal stability and resistance to water absorption properties. However, these performance on some applications have undesirable places, so we can use grafted cellulose to give it greater control. Functionality, intelligence and potential applications for the development of new bio-based materials, which have great significance to achieve a qualitative about the application of natural fibers from renewable resources[1].

Currently, grafted cellulose is mostly reacted in the emulsion, this preparation process is relatively complicated, and because the cellulose is not completely dissolved at this system, which make the reaction solution became a multi-phase syetem rsultiing in a low degree of cellulose and availability preparation

Heterogeneity and low value limit its application. Ionic liquids[2-3] as green solvents to dissolve cellulose can generate a clear solution and preparate product uniformity as a reaction medium and catalyst. So high value-added cellulose materials have gradually become a research focus[4].

During grafting polymerization, cellulose was carried out by traditional conductive heating. However, it is a comparatively inefficient method and time-consuming for transferring energy into the system. However, microwave irradiation can be directly and uniformly absorbed throughout the entire volume of an object and achieve the remarkable enhancement of the rates over conventional reaction. For instance, Lin[5] et al. reported a homogeneous modification poly Acrylic acid (AA) onto cotton linter by microwave irradiation. They found out that the reaction process was carried out in just three minutes and got a better yield of products.

The grafted copolymer (cellulose-g-PDMAEMA) was prepared with the use of potassium persulfate initiatoring radical polymerization process, which grafted functional monomer dimethylaminoethyl methacrylate(DMAEMA) on cellulose surface in the ionic liquid phase solution by microwave-assisted heating. During the preparation, which considered the crosslinking agent concentration, the concentration of initiator, monomer content, temperature and time factors and ultimately determined the optimal reaction conditions, which provide a new approach and guidance for bagasse cellulose grafted. 


\section{Experimental}

2.1 Materials. Sugarcane bagasse was provided from Guangdong province Country Harvest. 2-(Dimethylamino) ethylmethacrylate (DMAEMA, purity $\geq 99.9 \%$ ) was purchased from Sigma. All the other chemicals were of analysis grade.

2.2Homogeneoussynthesisofcellulose-g-PDMAEMA. The dissolution of cellulose was carried out according to the previous work [6].After completely dissolving the cellulose, the homogeneous solution was placed in a microwave reactor cellulose instrument, and added a certain amount of KPS, DMAEMA and MBA with a mixed solution of $5 \mathrm{ml}$ dimethylsulfoxide, which was heated under nitrogen protection by microwave irradiation $(400 \mathrm{~W} / 50 \mathrm{~Hz})$ to the reaction temperature until the reaction proceeds to the set time. When the reaction was complete, it was placed in a water bath of about $45^{\circ} \mathrm{C}$ where the reaction solution was added 5 volumes of deionized water to regenerate. After centrifugation, it was firstly washed with ethanol and then with acetone solution at $57^{\circ} \mathrm{C}$ soxhlet reflux for $24 \mathrm{~h}$, the precipitate was lastly centrifuged and dried to constant weight by vacuo.

During the process of microwave homogeneous reaction, the optimized conditions could be confirmed through analysis of grafting degree. The grafting rate was the mass change before and after grafting DMAEMA on the surface of bagasse cellulose, which was calculated on oven dry weight using the formula(Eq.1):

$$
Y=\frac{W_{\mathrm{g}}-W_{0}}{W_{0}} \times 100 \%
$$

Where $\mathrm{W}_{\mathrm{g}}$ is the amount of product after grafting, $\mathrm{W}_{\mathrm{o}}$ is the amount of the former grafted bagasse cellulose, $\mathrm{Y}$ is the graft ratio.

\section{Results and Discussion}

The polymerization was carried out with different crossing linking agent concentration ranging from $2.0 \%$ to $4.5 \%$ in Fig. 1 . It showed that the grafting rate increased with the increasing MBA concentration. Higher MBA concentration would promote more molecular weight DMAEMA "grafting" onto the surface of the cellulose, the opportunities of contacting with the reaction substance increased.

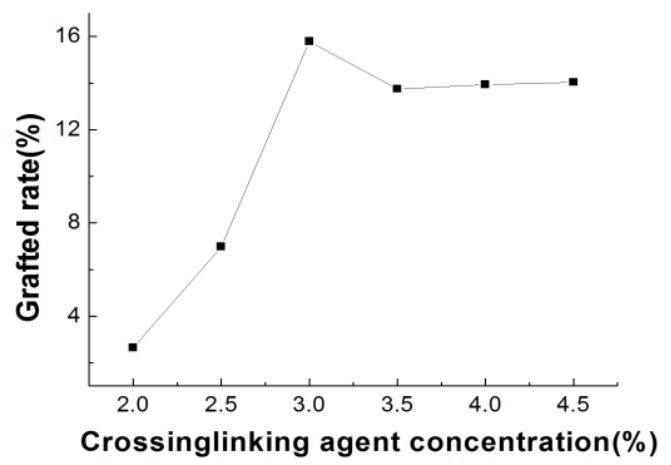

Fig. 1 The influence of crossinglinking concentration on grafting

The Fig. 2 shows the effect of initiator concentration on grafting. the grafting rate got the highest value when the concentration of KPS was $2.5 \%$, when the concentration of KPS was increased up to $2.5 \%$, the effective number of free radical increased, which speeding up the process, and thus the grafted rate improved. While, when the KPS concentration was continue added, KPS or the generated free radicals participated in coupling termination on the macromolecular chains of cellulose, and caused monomer homopolymerization resulting in the reduction of monomer conversion. 


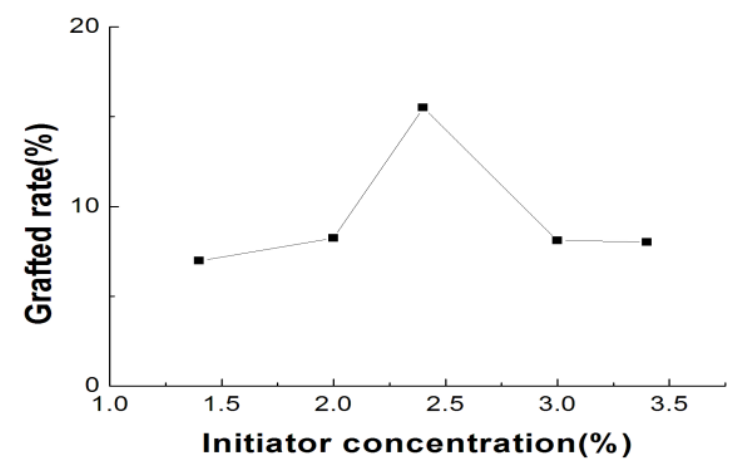

Fig.2 The influence of initiator concentration on grafting

The fig.3 showed grafting reaction was carried out with different concentration ratios, the grafting rate firstly increased and then decreased with the increase of the amount of monomer. The grafted rate was up to $15 \%$ when DMAEMA proportion increased to 1:5, which would help to improve the rate of polymerization and grafting rate. When the monomer ratio continued to increase, excessive monomer increased polymerization and the number of free-radical increased on homopolymer chains would be produced. Thus grafted rate showed a downward trend.

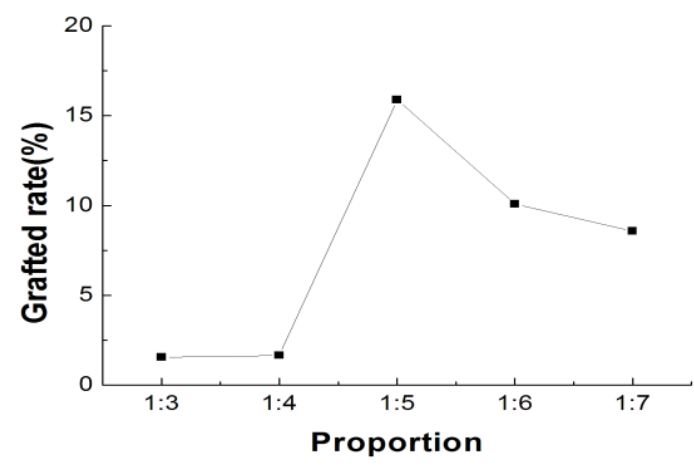

Fig. 3 The influence of monomer quantity on grafting

The fig. 4 showed the relationship between temperature and grafting rate. It was observed that grafting rate firstly increased and then decreased with the temperature increased. As the reaction temperature gradually increased, the rate of initiation of KPS would increase, and it could produce more free radicals as the temperature was too high to speed the reaction with grafting rate increasing. While the termination rate and homopolymerization would accelerate with monomer content increasing, the grafting rate turned to be decrease.

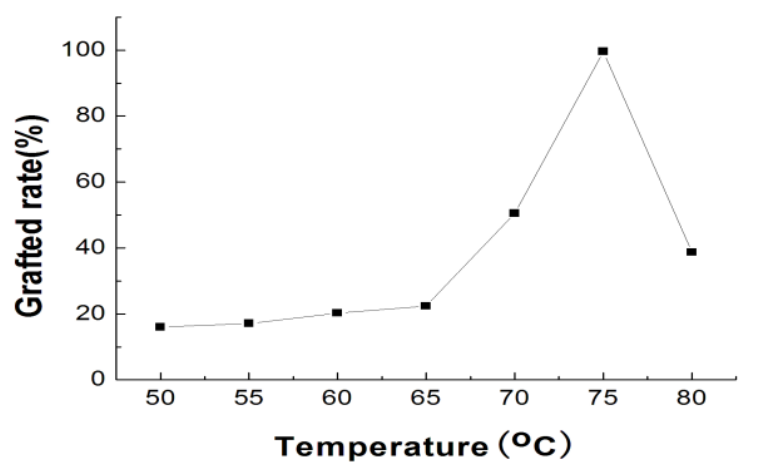

Fig.4 The influence of temperature on grafting

The fig. 5 showed the different grafted rate with different reaction time. The grafting rate showed a gradual increasing trend with the growth of time. Firstly the graft polymerization rate was not fast due to less number of free radicals generated by oxidation of KPS as the reaction started. then a sufficient number of free radicals were generated with time increased, which accelerated the radicals active contact between monomer and cellulose macromolecules, thereby the grafting rate 
increased accordingly with the reaction rate of the graft polymerization.

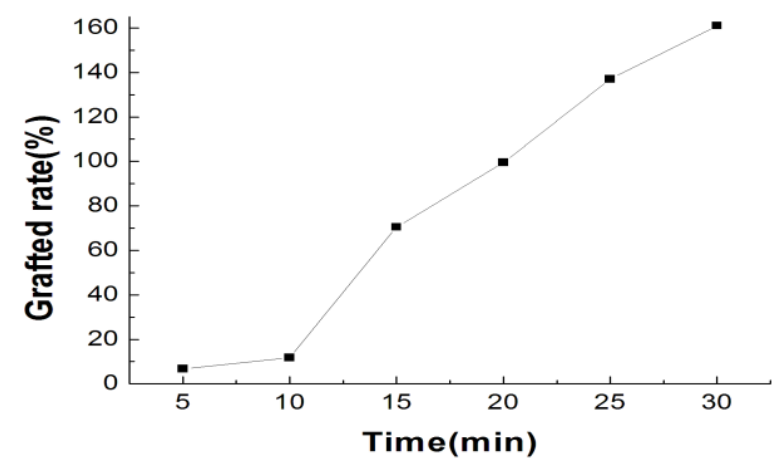

Fig.5 The influence of reaction time on grafting

\section{Characterization}

FT-IR spectroscopy and XRD were carried out to characterize the grafted cellulose copolymer.

Fourier transform infrared spectroscopy (FTIR), which Spectrum GX-1 used to test the cellulose-g-PDMAEMA and bagasse cellulose prepared powders after grinding with $\mathrm{KBr}$ tablet, a resolution of $4 \mathrm{~cm}^{-1}$, wave number range of $4000-400 \mathrm{~cm}^{-1}$ and 8 times of the frequency.

Jappan DLMAX-2550 neo diffractometer tested materials crystal, which weighed $0.1 \mathrm{~g}$ cellulose-g-PDMAEMA and bagasse cellulose, testing voltage was $40 \mathrm{KV}, \mathrm{Cu}$ target $\mathrm{Ka}$ radiation was $\mathrm{k}=1.4056 \AA$, $2 \theta$ testing ranges were $10^{\circ}$ to $80^{\circ}$, the sanning speed of $6^{\circ} / \mathrm{min}$.

Fig. 6 shows the FT-IR spectra of the non-grafted cellulose and the grafted copolymer samples. Compared with the spectrum of the non-grafted cellulose, the peak at $1730 \mathrm{~cm}^{-1}$ was indicated that the grafted cellulose was assigned to the characteristic stretching vibrations of $\mathrm{C}=\mathrm{O}$ in the carbonyl group of polymer chains pDMAEMA. The appearance of this peak has been proved the grafting of polymer chains onto the surface of cellulose.

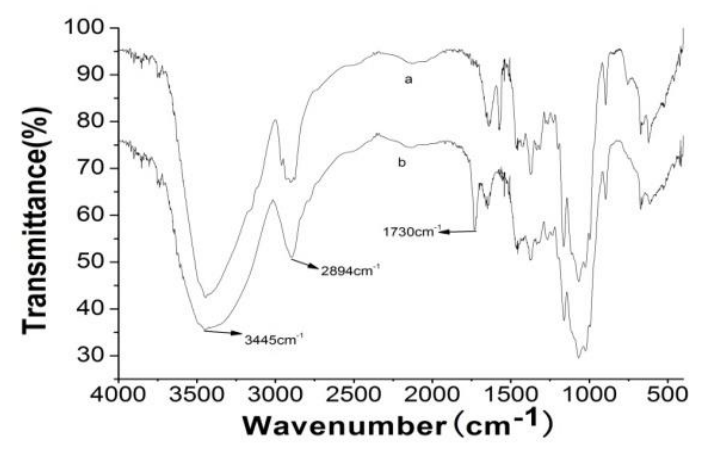

Fig.6 FTIR spectra of non-grafted cellulose(a)and grafted cellulose(b)

The XRD diffraction profile of two samples was also evaluated, shown in Fig.7. Compared with the non-grafted cellulose, these native cellulose type II peaks of $12^{\circ}, 20^{\circ}$ and $22^{\circ}$, while grafted cellulose gradually disappeared due to the destroy of characteristic peak of cellulose and the crystal morphology was destroyed 


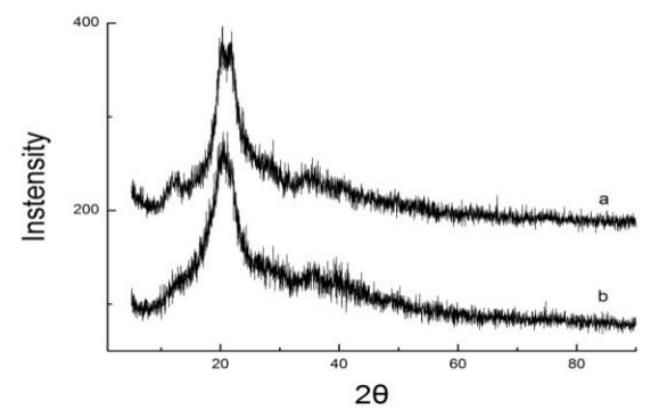

Fig.7 XRD patterns of non-grafted cellulose (a) and grafted cellulose(b)

Establishment of polymerization kinetic equation

\section{Conclusions}

The grafted copolymer cellulose-g-PDMAEMA was prepared by microwave-assisted in a homogeneous system. The preparation investigated MBA concentrations, KPS concentration, the amount of monomer, reaction temperature and time on the grafting process, and obtained optimum preparation conditions: G:D was 1:5,KPS concentration of $2.4 \%$, MBA concentration of $3 \%$, the reaction time was $20 \mathrm{~min}$, the reaction temperature was $75^{\circ} \mathrm{C}$, the grating ratio could be close to $100 \%$, it shows that microwave-assisted leaded can accelerate the reaction rate.

\section{Acknowledgements}

We gratefully acknowledge the Fundamental Scientific Research Funds for Chinese Academy of Tropical Agricultural Sciences (No.1630062015020) and (No.1630062013012). The work is also partially supported by the Natural Science Foundation of Hainan Province (20155204).

\section{References}

1. Huaiyu Zhan. Science Press, Beijing, China, (2005).

2. C Iee. US, 6129867,(2000).

3. IA Soga, RH Atalla. Cellulose, 5:309-319,(1998).

4. Jun Ye, Xingfei Zhao, Jian Xiong. Progress in Chenistry, 19(4):478-484,(2007).

5. C.X. Lin, H.Y. Zhan, M.H. Liu, S.Y. Fu and L.H. Huang. Appl. Polym. Sci. Vol.118 , p.399,(2010).

6. J.H. Li, Y.F. Tang, J.C. Chen, Y.P. Cao, M.F. Huang, T.K. Fu and J.B. Su. Adv. Mater. Res. Vol.152-153, p. 1570,(2011). 